\title{
Incorporating Missing Outcome Data in The Sample Size Calculation For a Future Trial: A Case Study Using a Single Trial, a Pairwise and Network Meta- Analysis
}

Loukia Spineli ( $\nabla$ spineli.loukia@mh-hannover.de)

Hannover Medical School

\section{Research Article}

Keywords: Systematic review, Missing outcome data, Network meta-analysis, Sample size, Clinical trial, Absolute risks

Posted Date: February 16th, 2021

DOI: https://doi.org/10.21203/rs.3.rs-198337/v1

License: (c) (1) This work is licensed under a Creative Commons Attribution 4.0 International License. Read Full License 
1 Incorporating missing outcome data in the sample size calculation for a

2 future trial: a case study using a single trial, a pairwise and network meta3 analysis

4

$5 \quad$ Loukia M. Spineli Spineli.Loukia@mh-hannover.de

6 Midwifery Research and Education Unit, Hannover Medical School, Hannover,

7 Germany

8

9 Corresponding author: Loukia M. Spineli, Midwifery Research and Education

10 Unit (OE 9210), Hannover Medical School, Carl-Neuberg-Straße 1, 30625,

11 Hannover, Germany.

12 E-mail: Spineli.Loukia@mh-hannover.de

13 Telephone: +49 (0) $511532-83593$ 


\section{$1 \quad$ Abstract}

2 Background: To illustrate the advantages of using network meta-analysis (NMA) as

3 compared to a trial or a pairwise meta-analysis to estimate the amount of missing outcome

4 data (MOD) for a target comparison in order to adjust the required sample size for possible

5 participant losses in a future trial.

6 Methods: We introduced the concept of transitive risks to obtain the absolute risks of MOD

7 for all interventions of the network. We used the network of a published systematic review on

8 a binary outcome to apply the proposed concept and to calculate the required sample size in a

9 future trial for a selected target comparison. For that comparison, we also calculated the required sample size using the corresponding trials separately, and after pooling these trials in a random-effects meta-analysis.

Results: Ignoring MOD from the sample size calculation led to the smallest sample size.

When either trial was considered, the risk of MOD ranged from $1 \%$ to $13 \%$ in the compared intervention arms, therefore, increasing the sample size from $1 \%$ to $12 \%$. Performing a pairwise meta-analysis yielded a risk of MOD equal to $6 \%$ and $9 \%$ in the active and control arms, respectively, which inflated the sample size by $8 \%$. Using NMA, the corresponding risks of MOD were $10 \%$ and $13 \%$, which increased the sample size by $13 \%$.

Conclusions: Provided that the transitivity assumption holds, incorporating the absolute risks of MOD in the sample size calculation for a target comparison of the network led to better planning of a future trial.

Keywords: Systematic review; Missing outcome data; Network meta-analysis; Sample size; Clinical trial; Absolute risks. 


\section{Background}

2 Patient dropouts are ubiquitous in clinical trials for various reasons, some of which may be

3 related to the design and conduct of the trial. Knowledge of the number of and reasons for

4 missing outcome data (MOD) is necessary to design a clinical trial that will deliver results of

5 high quality [1]. Information on the number of missing participants in the compared

6 interventions will ensure a sufficient sample size to detect the minimum clinically relevant

7 difference for a specific outcome and target population. Information on the possible reasons

8 for MOD will lead to a proactive design of the future clinical trial to retain most of the

9 randomised participants, and hence, increase statistical power [1].

Use of evidence synthesis to design a clinical trial has been long advocated as the key against research and funding waste because it can reveal limitations in the conduct and analysis of past relevant trials to prioritise future research [2]. The research community holds a positive attitude to a research agenda for future trials based on the synthesis of past relevant trials $[3,4]$. Evidence synthesis can also play a crucial role in uncovering the extent of MOD in a specific patient population, set of interventions and outcome under investigation. Applications of pairwise and network meta-analysis have revealed that trials with the same patient population and intervention setting encountered variability on the amount of MOD [57]. Selecting any of these past trials to inform the sample size of a relevant future trial would be misleading. This is because failure to incorporate the variability in the amount of MOD (as observed in a series of relevant trials) in the design of a future trial may result in an underpowered trial, thus compromising any efforts to create an ecosystem of the best available evidence.

There is already published methodology on the planning of future trials based on systematic reviews [8-10]. However, this methodology does not take into account the effect 
of MOD on the sample size calculations for a future trial. Considering a fictional two-arm trial for illustration, Cook and Zea [11] performed a sensitivity analysis to investigate how the amount of MOD and different assumptions about the missingness mechanisms in the compared arms can influence the sample size calculations for a future trial. The authors demonstrated that the required sample size to achieve the desired power was dramatically larger when the calculations were based on the sensitivity analysis as compared to ignoring MOD altogether [11]. To the best of our knowledge, there is currently no research at the level of evidence synthesis to demonstrate the implications on sample size calculations when MOD from a series of relevant past trials are incorporated into the design of a future trial.

It is legitimate to wonder about the 'proper' synthesis framework to inform the future trial: pairwise or network meta-analysis. A pairwise meta-analysis restricts the synthesis of trials to one comparison, therefore, providing only a fraction of the 'underlying' extent of MOD in the compared interventions. Arguably, network meta-analysis (NMA) is the ideal framework to base the planning of future trials on, as it synthesises a series of relevant trials investigating a different set of interventions. Furthermore, it can inform decisions for comparisons never investigated in a trial. Therefore, NMA can be used to prioritise the target comparison(s) to be investigated in the future trial(s) [9]. In the context of MOD, NMA can maximise the information on MOD contained in different sets of interventions across trials in order to provide a sufficient sample size for a future trial on a specific comparison. Ergo, by taking into account the variability in the amount of MOD within the network, we can adjust the required sample size for the expected participant losses in the future trial for a target comparison.

The present study aims to illustrate the advantages of using NMA rather than a trial or a pairwise meta-analysis to estimate the amount of MOD for a target comparison in order to 
1 adjust the required sample size for possible participant losses in a future trial. For that

2 purpose, we introduce the concept of transitive risks across trials to estimate the risk of MOD

3 for each intervention within the NMA framework [12]. We use a published systematic review

4 as a motivating example to illustrate the concept of transitive risks and the implications of

5 MOD on the sample size calculation for a future trial.

\section{Methods}

7 As a motivating example, we used the systematic review of Baker et al. [13] for the treatment 8 of chronic obstructive pulmonary disease (COPD) exacerbations (Table S1 in Additional file

9 1). Figure $1 \mathrm{~A}$ illustrates the network of five interventions alongside the extent of MOD in each intervention and observed comparison: the percentage of MOD (\%MOD) signified moderate (more than $5 \%$ and up to $20 \%$ ) and high attrition bias (>20\%) within the network. According to Table 1, \%MOD varied substantially in all interventions ranging from low $(\leq$ $5 \%)$ to moderate in tiotropium and high in the remaining interventions. The \% MOD also varied considerably within the majority of the observed comparisons (Table 1).

\section{[Table 1]}

For illustrative purposes, we focused on the comparison of tiotropium with long-acting $\beta_{2}-$ agonist (LABA) that was investigated in three clinical trials (Table S2 in Additional file 1). For this comparison, the publication reported an odds ratio (OR) of 0.82 (95\% credible interval (CrI): 0.72-0.93) in favour of tiotropium (Figure $2 \mathrm{~A}$ in [13]). However, it is not explicit in the report how MOD were addressed in the NMA model. Therefore, we reanalysed the network while modelling MOD under the missing at random (MAR) assumption to acknowledge the uncertainty induced by MOD and maintain the randomised sample [7]. 
2

3

4

\section{Transitive risks assumption}

To be able to introduce the concept of transitive risks, we have assumed the transitivity assumption and its statistical manifestation (consistency) are plausible; otherwise, the transitive risks would be invalid [12]. The concept of transitive risks builds upon the transitivity assumption; namely, trials share similar clinical and methodological characteristics, and differ only in the interventions compared [12]. In essence, the assumption of transitive risks implies that the risk of the outcome in an intervention (hereafter, absolute risk) is similar across all trials of the network. In other words, an intervention is assumed to have an exchangeable absolute risk across all trials irrespective of the comparator intervention(s) in each trial [12]. This notion of transitive risks stems from the interpretation of the transitivity assumption in Salanti [14]: 'There are no differences between observed and unobserved relative effects of AC and BC beyond what can be explained by heterogeneity'.

While the assumption of transitive risks might seem difficult to defend in practice, it facilitates the estimation of unique absolute risks for each intervention [12]. Specifically, for the calculation of the absolute risks, we only need (i) the estimated relative treatment effects from NMA (e.g. $\log$ OR) for comparisons with the selected reference intervention of the network, and (ii) a sensible assumption about the underlying risk in the reference intervention [12]. Below we illustrate the calculation of the absolute risks following the GRADE concept for binary outcomes [15]. We used the Bayesian framework to estimate the (posterior) median and $95 \% \mathrm{CrI}$ of the absolute risk for each intervention.

\section{Absolute risks under the assumption of transitive risks}


1 With $d_{k}$ we indicate the posterior mean of the $\log$ OR between intervention $k$ ( $k=$

$2 B, C, \ldots, T)$ and reference intervention $A$. With $p_{A}$ we imply the underlying risk for the

3 reference intervention that has been selected ideally from observational studies or relevant

4 randomised trials (in the absence of the former) [15]. The GRADE approach advocates the

5 risk ratio (RR) as a measure of the relative effect. However, we used the OR for its statistical

6 advantages and for not requiring constraints to ensure that the probability of an event is within

$7(0,1)-$ as opposed to RR [16]. Following Walter [17], we obtain the absolute risk in each

8 intervention as a function of $d_{k}$ and $p_{A}$ :

$$
p_{k}=\frac{\exp \left(d_{k}\right) p_{A}}{1+p_{A}\left(\exp \left(d_{k}\right)-1\right)}
$$

9 Note that equation (1) is equivalent to the predicted risk for intervention $k$ as described by 10 Dias et al. [18],

$$
\operatorname{logit}\left(p_{k}\right)=\operatorname{logit}\left(p_{A}\right)+d_{k}
$$

11 where $\operatorname{logit}\left(p_{A}\right)$ is the log odds of an event in the reference intervention.

\section{Illustration of obtaining absolute risk assuming transitive risks}

Figure $1 \mathrm{~B}$ presents the network of four types of counselling for smoking cessation (no contact, self-help, individual counselling, and group counselling) [19]. We considered this network to illustrate the estimation of the absolute risk of success (smoking cessation) in each intervention under the assumption of transitive risks (Table S3 in Additional file 1). For illustration, we considered an $8 \%$ underlying risk of abstinence from smoking in 'no contact' (the reference intervention) which is the median risk across the trials that included that intervention. We performed Bayesian random-effects NMA with the incorporation of the 
equation (1) in the model. Information on the model implementation is found in Additional file 2.

Table 2 illustrates the estimated OR (posterior mean and 95\% CrIs) for all comparisons with 'no contact' and the absolute risks for each intervention under the assumption of transitive risks. Group counselling was associated with the highest rate of smoking cessation (0.20 with $95 \%$ CrI: $0.10,0.38)$, followed by individual counselling $(0.17$ with $95 \%$ CrI: 0.11 , $0.25)$ and self-help $(0.12$ with $95 \%$ CrI: $0.06,0.24)$. Note the wide width of $95 \% \mathrm{CrI}$ in the absolute risks of all interventions, which may be partly attributed to the substantial betweentrial variance (posterior median $0.79,95 \%$ CrI: $0.54,1.21$ ).

[Table 2]

\section{Sample size adjustments for MOD in a future two-arm trial}

We plan a future two-arm trial, and we assume an event risk of $p_{1}$ and $p_{2}$ for the control and experimental intervention, respectively. We use a Z-test to assess the null hypothesis of no difference (i.e. $H_{0}: p_{2}-p_{1}=0$ ) against the two-sided alternative hypothesis (i.e. $H_{A}: p_{2}-$ $p_{1} \neq 0$ ). Under $H_{0}, p_{2}-p_{1}$ is assumed to follow a normal distribution with zero mean and variance $2 \bar{p}(1-\bar{p}) / n$ where $\bar{p}=\left(p_{1}+p_{2}\right) / 2$. Under $H_{A}, p_{2}-p_{1}$ follows a normal distribution with mean $\Delta \neq 0$ and variance $\left[p_{1}\left(1-p_{1}\right)+p_{2}\left(1-p_{2}\right)\right] / n$. For a pre-specified power at $1-\beta$, and type I error at $a$, the required sample size in the absence of MOD is

$$
n=\left(\frac{2 Z_{1-a / 2} \sqrt{\bar{p}(1-\bar{p})}+Z_{1-\beta} \sqrt{2 p_{1}\left(1-p_{1}\right)+2 p_{2}\left(1-p_{2}\right)}}{p_{2}-p_{1}}\right)^{2}
$$

where $Z_{q}$ is the $\mathrm{q}^{\text {th }}$ percentile of the standard normal distribution [20]. 
When we expect MOD in the future trial, under the MAR assumption, the required sample

2 size in the future trial with $1: 1$ randomisation is inflated by $1 /\left(1-q_{k}\right)$ where $q_{k}$ is the

3 probability of MOD in arm $k$. Then the required sample size to achieve a power of $1-\beta$ after

4 adjusting for MOD is

$$
n^{*}=\sum_{k=1}^{2} \frac{n}{2}\left(\frac{1}{1-q_{k}}\right)
$$

\section{$5 \quad$ Implementation for the motivating example}

6 We performed Bayesian random-effects NMA while modelling MOD under the MAR 7 assumption [7] to adjust for MOD properly, and we incorporated equation (1) into the model 8 to obtain the absolute risks $p_{1}$ and $p_{2}$ for LABA and tiotropium, respectively, while assuming $9 \quad p_{A}=0.39$ in placebo (median observed event risk across the placebo-controlled trials). We used the estimated $p_{1}$ and $p_{2}$ in equation (3) to calculate the required sample size before adjusting for the expected MOD in LABA and tiotropium in the future trial. We considered $80 \%$ power and $5 \%$ type I error.

Subsequently, for each trial, we calculated $q_{1}$ and $q_{2}$ as the ratio of MOD to the number randomised in each arm. At the pairwise meta-analysis level, we pooled the three trials that compared tiotropium with LABA for the dropouts outcome using the Bayesian randomeffects meta-analysis model as described by Dias et al. [21] to obtain $q_{2}$ assuming $q_{1}=0.09$ (median event risk in LABA). For the NMA, we used the whole network (Figure 1A) and we worked in line with the pairwise meta-analysis to obtain $q_{1}$ and $q_{2}$ for LABA and tiotropium, respectively, while assuming $q_{A}=0.18$ in placebo (median risk of MOD across the placebocontrolled trials). Finally, we applied equation (4) to obtain the adjusted sample size for that 
1 comparison at (i) the trial level, (ii) pairwise meta-analysis level, and (ii) network meta-

2 analysis level. Information on the technical details of the models is found in Additional file 2.

\section{$3 \quad$ Results}

\section{$4 \quad$ Absolute risks of the primary outcome (COPD exacerbations)}

5 Figure 2 illustrates the absolute risks of COPD exacerbations for all interventions in the

6 network under the assumption of transitive risks. The posterior median (and 95\% CrI) of the

7 absolute risk of COPD exacerbations for LABA and tiotropium were $p_{1}=0.31$ (95\% CrI:

$8 \quad 0.24-0.39)$ and $p_{2}=0.27$ (95\% CrI: $\left.0.21-0.34\right)$, respectively. After adjusting for MOD,

9 the posterior mean of the OR of COPD exacerbations in tiotropium versus LABA was 0.84 (95\% CrI: $0.57-1.18)$ which was similar to the reported OR in the publication: $0.82(95 \%$ CrI: $0.72-0.93)$. However, our results failed to demonstrate conclusive evidence in favour of

tiotropium due to substantial missingness in the network. Using equation (3), the total unadjusted required sample size for a future trial on tiotropium versus LABA was $n=4038$ (or 2019 participants per arm).

\section{[Figure 2]}

\section{Total sample size adjusted for missing outcome data}

Figure 3A summarises the adjusted and unadjusted sample size calculations for the three different levels of evidence. Figure 3B illustrates the corresponding absolute risk of MOD per intervention. At the trial level, $q_{1}$ and $q_{2}$ for LABA and tiotropium, respectively, were 0.09 and 0.05 in trial one, 0.13 and 0.09 in trial two, and 0.01 and 0.01 in trial three (Figure 3B).

Then, using equation (4) for $n=4038$, the total required sample size after adjusting for MOD in the compared arms was 4344, 4539, and 4079 in the corresponding trials (Figure 
$13 \mathrm{~A})$. Incorporating MOD in the sample size calculation inflated the required sample size by

$28 \%, 12 \%$, and $1 \%$ in the corresponding trials. The inflation varied across the trials: trials two

3 and three received the highest and lowest inflation in the sample size calculation, respectively,

4 for having the most and least MOD in the compared arms.

[Figure 3]

At the meta-analysis level, for $q_{1}=0.09$ (assumed) and $q_{2}=0.06$ (posterior median with 95\% CrI: $0.03-0.10)$, the total required sample size after adjusting for MOD in LABA and tiotropium was $n^{*}=4367$ which was $8 \%$ larger than $n=4038$ (Figure 3A). Using NMA, the posterior median of $q_{1}$ and $q_{2}$ were 0.13 (95\% CrI: $\left.0.11-0.15\right)$ and 0.10 (95\% CrI: $0.08-$ 0.12), respectively, for LABA and tiotropium (Figure 3B). Then, using equation (4), the total required sample size after adjusting for MOD was $n^{*}=4564$ (Figure 3A) which was $13 \%$ larger than $n=4038,5 \%$ larger than $n^{*}=4367$ in the pairwise meta-analysis, and $1 \%$ larger than $n^{*}=4539$ in trial 2.

\section{Discussion}

This is the first study to illustrate the gains of using NMA instead of a single trial or pairwise meta-analysis to calculate the sample size for a future trial while accounting for expected MOD. With the present work, we aimed to contribute to the growing literature on the importance of NMA in the planning of future trials [22,23] by demonstrating another advantage of NMA to this direction: maximising the information on the extent of MOD to estimate a sufficient sample size for a future trial. Being an inevitable challenge in the conduct and analysis of a clinical trial, MOD should be an integral part of the considerations for the design of a new trial. We illustrated that individual trials and pairwise meta-analysis reflected only a fragment of the extent of MOD for a specific set of interventions and target 
1 patient population. Reliance on either evidence to calculate the required sample size led to an

2 underpowered future trial. The implications were also profound when MOD were not

3 considered in the sample size calculation.

$4 \quad$ We acknowledge that we used a simplified framework to select the target comparison and

5 perform the sample size calculation. This is the main limitation of our study. In real-life

6 practice, the costs, treatment availability, and priority for the development of clinical

7 guidelines are among the factors that affect the selection of the target comparison for a future

8 trial $[9,22]$. Furthermore, considerations on the optimal design of a future study to provide

9 conclusive results include sophisticated methods which are extensions of established methods for clinical trials (e.g. [22,24]). Notably, the design of a future trial is the product of the joint effort of a multidisciplinary group of experts, and is based on regulated processes. Nevertheless, we expect the implications of MOD on the sample size calculation for a future trial, as illustrated in the present study, to be fundamentally the same, regardless of the framework.

Furthermore, we considered only one network to illustrate the merits of incorporating MOD from a series of trials into the sample size calculations. An empirical study on a collection of networks from several health fields would demystify the degree of underestimation in the sample size for a new trial when MOD are not accounted for in the design. We acknowledge that an extensive collection of networks with complete information on MOD for each intervention arm of every trial would be a challenging endeavour, as recent evidence on the reporting quality of systematic reviews concerning MOD has been underwhelming $[25,26]$.

The findings of the present study revealed the importance of incorporating MOD into the sample size calculation. However, the ubiquity of MOD in all levels of evidence renders 
necessary the proper handling of MOD in the analysis. We view the collaboration between statisticians and clinicians as imperative for the successful handling of MOD, especially, at the level of systematic reviews where the extent of MOD is variable across the trials [7,27] and information about the characteristics of missing participants are not available without access to individual patient data [28]. In the light of these limitations, a panel of expert clinicians on the target condition and interventions is crucial for determining the plausible scenarios about the MOD mechanisms. Then, a team of statisticians can integrate these scenarios into a modelling framework (e.g. pattern-mixture model $[7,29]$ ) to adjust the NMA results for imminent attrition bias.

\section{Conclusions}

We regard the concept of the 'conditional trial design' proposed by Salanti et al. [23] as a promising framework to promote a healthy evidence ecosystem. Our proposed framework can be incorporated straightforwardly to the stepwise process of the conditional trial design [23]. Initially, the NMA model is extended to encompass the model for MOD to yield internally coherent relative treatment effects that are adjusted for potential attrition bias (step 1) [7,29]. After deciding on the target comparison(s) (step 2), and the evidence from the NMA is conclusive (step 3), the concept of transitive risks is used to estimate the absolute risk of MOD in the interventions of the target comparison(s). Then, these absolute risks of MOD are included in the sample size calculation for the new trial (step 4).

\section{Abbreviations}

COPD: chronic obstructive pulmonary disease; CrI: credible intervals; LABA: long-acting 32-agonist; MAR: missing at random; MOD: missing outcome data; \%MOD: percentage of missing outcome data; NMA: network meta-analysis; OR: odds ratio; RR: risk ratio. 


\section{Declarations}

\section{Ethics approval and consent to participate}

3 Not applicable.

\section{Consent for publication}

$5 \quad$ Not applicable.

\section{$6 \quad$ Availability of data and materials}

7 All data analysed during this study are included in this article and its supplementary 8 information files.

\section{Competing interests}

10 The author declares no competing interests.

\section{Funding}

13 Forschungsgemeinschaft under grant number SP 1664/1-3). The funder had no involvement in

14 study design; in the collection, analysis, and interpretation of data; in the writing of the report;

15 and in the decision to submit the article for publication.

\section{Authors' contributions}

17 LMS conceived and designed the study; acquired, analysed and interpreted the data; drafted and revised the manuscript. The author read and approved the final manuscript.

\section{Acknowledgements}


1 The author would like to thank Dr Joanne Welsh for commenting on an earlier version of the

2 article.

\section{$3 \quad$ References}

4 1. Hughes S, Harris J, Flack N, Cuffe RL. The statistician's role in the prevention of missing data. Pharm Stat. 2012;11(5):410-6.

2. Sutton AJ, Cooper NJ, Jones DR. Evidence synthesis as the key to more coherent and efficient research. BMC Med Res Methodol. 2009;9:29.

3. Clayton GL, Smith IL, Higgins JPT, Mihaylova B, Thorpe B, Cicero R, et al. The INVEST project: Investigating the use of evidence synthesis in the design and analysis of clinical trials. Trials. 2017;18(1):219.

4. Nikolakopoulou A, Trelle S, Sutton AJ, Egger M, Salanti G. Synthesizing existing evidence to design future trials: survey of methodologists from European institutions. Trials. 2019; 20(1):334.

5. Higgins JP, White IR, Wood AM. Imputation methods for missing outcome data in meta-analysis of clinical trials. Clin Trials. 2008;5(3):225-39.

6. Spineli LM. Modeling missing binary outcome data while preserving transitivity assumption yielded more credible network meta-analysis results. J Clin Epidemiol. 2019;105:19-26.

7. Spineli LM. An empirical comparison of Bayesian modelling strategies for missing binary outcome data in network meta-analysis. BMC Med Res Methodol. 2019;19(1):86. 
1 8. Nikolakopoulou A, Mavridis D, Salanti G. Using conditional power of network metaanalysis (NMA) to inform the design of future clinical trials. Biom J. 2014;56(6):97390.

9. Nikolakopoulou A, Mavridis D, Salanti G. Planning future studies based on the precision of network meta-analysis results. Stat Med. 2016;35(7):978-1000.

10. Roloff V, Higgins JP, Sutton AJ. Planning future studies based on the conditional power of a meta-analysis. Stat Med. 2013;32(1):11-24.

11. Cook T, Zea R. Missing data and sensitivity analysis for binary data with implications for sample size and power of randomized clinical trials. Stat Med. 2020;39(2):192-204.

12. Spineli LM, Brignardello-Petersen R, Heen AF, Achille F, Brandt L, Guyatt GH, et al. Obtaining absolute effect estimates to facilitate shared decision making in the context of multiple-treatment comparisons. Global Evidence Summit, 2017. dx.doi.org/10.1002/14651858.CD201702.

13. Baker WL, Baker EL, Coleman CI. Pharmacologic treatments for chronic obstructive pulmonary disease: a mixed-treatment comparison meta-analysis. Pharmacotherapy. 2009;29(8):891-905.

14. Salanti G. Indirect and mixed-treatment comparison, network, or multiple-treatments meta-analysis: many names, many benefits, many concerns for the next generation evidence synthesis tool. Res Synth Methods. 2012;3(2):80-97.

15. Guyatt GH, Oxman AD, Santesso N, Helfand M, Vist G, Kunz R, et al. GRADE guidelines: 12. Preparing summary of findings tables-binary outcomes. J Clin Epidemiol. 2013;66(2):158-72. 
1 16. Bakbergenuly I, Hoaglin DC, Kulinskaya E. Pitfalls of using the risk ratio in metaanalysis. Res Synth Methods. 2019;10(3):398-419.

17. Walter SD. Choice of effect measure for epidemiological data. J Clin Epidemiol. 2000;53(9):931-9.

18. Dias S, Welton NJ, Sutton AJ, Ades AE. NICE DSU Technical Support Document 2: A Generalised Linear Modelling Framework for Pairwise and Network Meta-Analysis of Randomised Controlled Trials. London: National Institute for Health and Care Excellence (NICE); April 2014.

19. Fiore MC, Bailey WC, Cohen SJ, et al. Smoking Cessation. Clinical Practice Guideline No. 18. Rockville, MD: Agency for Health Care Policy and Research, U.S. Department of Health and Human Services, AHCPR Publication No 96-0692, April 1996.

20. Ciarleglio MM, Arendt CD. Sample size determination for a binary response in a superiority clinical trial using a hybrid classical and Bayesian procedure. Trials. 2017;18(1):83.

21. Dias S, Sutton AJ, Ades AE, Welton NJ. Evidence synthesis for decision making 2: a generalized linear modeling framework for pairwise and network meta-analysis of randomized controlled trials. Med Decis Making. 2013;33(5):607-17.

22. Nikolakopoulou A, Mavridis D, Furukawa TA, Cipriani A, Tricco AC, Straus SE, et al. Living network meta-analysis compared with pairwise meta-analysis in comparative effectiveness research: empirical study. BMJ. 2018;360:k585.

23. Salanti G, Nikolakopoulou A, Sutton AJ, Reichenbach S, Trelle S, Naci H, et al. Planning a future randomized clinical trial based on a network of relevant past trials. 
Trials. 2018;19(1):365.

24. Nikolakopoulou A, Mavridis D, Egger M, Salanti G. Continuously updated network meta-analysis and statistical monitoring for timely decision-making. Stat Methods Med Res. 2018;27(5):1312-30.

25. Spineli LM, Yepes-Nuñez JJ, Schünemann HJ. A systematic survey shows that reporting and handling of missing outcome data in networks of interventions is poor. BMC Med Res Methodol. 2018;18(1):115.

26. Kahale LA, Diab B, Brignardello-Petersen R, Agarwal A, Mustafa RA, Kwong J, et al. Systematic reviews do not adequately report or address missing outcome data in their analyses: a methodological survey. J Clin Epidemiol. 2018;99:14-23.

27. Kahale LA, Khamis AM, Diab B, Chang Y, Lopes LC, Agarwal A, et al. Potential impact of missing outcome data on treatment effects in systematic reviews: imputation study. BMJ. 2020;370:m2898.

28. National Research Council. The Prevention and Treatment of Missing Data in Clinical Trials Panel on Handling Missing Data in Clinical Trials. Committee on National Statistics, Division of Behavioral and Social Sciences and Education. Washington, DC: The National Academies Press. 2010.

29. Mavridis D, White IR, Higgins JP, Cipriani A, Salanti G. Allowing for uncertainty due to missing continuous outcome data in pairwise and network meta-analysis. Stat Med. 2015;34(5):721-41. 


\section{Tables}

2 Table 1. Distribution of percentage missing outcome data in the network

\begin{tabular}{lcccccc}
\hline \multicolumn{1}{c}{ Intervention } & Trials & Min. & $1^{\text {rst }}$ qrtl. & Median & $3^{\text {nd }}$ qrtl. & Max. \\
\hline PBO & 19 & 5 & 13 & 18 & 22 & 44 \\
LABA & 14 & 1 & 10 & 14 & 21 & 32 \\
ICS & 5 & 0 & 13 & 27 & 31 & 40 \\
ICS+LABA & 5 & 5 & 12 & 28 & 30 & 32 \\
TIO & 7 & 1 & 5 & 8 & 9 & 12 \\
\hline \multicolumn{1}{c}{ Comparison } & Trials & Min. & $1^{\text {rst }}$ qrtl. & Median & $3^{\text {nd }}$ qrtl. & Max. \\
\hline LABA $v s$. PBO & 12 & 5 & 13 & 18 & 26 & 38 \\
ICS $v s$. PBO & 5 & 6 & 16 & 29 & 38 & 39 \\
ICS $v s$. LABA & 3 & 29 & 31 & 32 & 33 & 34 \\
ICS+LABA $v s$. PBO & 5 & 6 & 9 & 31 & 35 & 36 \\
ICS+LABA $v s$. LABA & 4 & 3 & 23 & 30 & 30 & 31 \\
ICS+LABA $v s$. ICS & 3 & 28 & 29 & 30 & 33 & 36 \\
TIO $v s$. PBO & 5 & 6 & 10 & 11 & 13 & 16 \\
TIO $v s$. LABA & 3 & 1 & 4 & 7 & 9 & 11 \\
\hline
\end{tabular}

3 Note: ICS, inhaled corticosteroid; LABA, long-acting $\beta 2$-agonist; Max, maximum; Min,

4 minimum; PBO, placebo; qrtl, quartile; TIO, tiotropium.

5

6 Table 2. Absolute risks using the assumption of transitive risks

\begin{tabular}{lcc}
\hline \multicolumn{1}{c}{ Comparison with } & OR $(95 \%$ CrI $)$ & $p_{k}(95 \%$ CrI $)$ \\
\hline No contact & - & 0.08 \\
self-help & $1.76(0.76,3.59)$ & $0.12(0.06,0.24)$ \\
individual counselling & $2.38(1.49,3.74)$ & $0.17(0.11,0.25)$ \\
group counselling & $3.28(1.31,7.16)$ & $0.20(0.10,0.38)$ \\
\hline Posterior median of between-trial variance: $0.79(95 \%$ CrI: $0.54,1.21)$
\end{tabular}

7 Posterior median of between-trial variance: 0.79 (95\% CrI: $0.54,1.21)$ 


\section{Figure legends}

Figure 1. A network of interventions for the maintenance treatment of the exacerbations of chronic obstructive pulmonary disease (network A) [13]. The different colours in the legend refer to the median percentage of missing outcome data across the trials. A network of four types of counselling for smoking cessation (network B) [19]. In both networks, the size of the nodes is proportional to the number of direct treatment comparisons that include that node, and the thickness of the links is proportional to the number of trials investigating the corresponding comparisons. ICS, inhaled corticosteroid; LABA, long-acting $\beta 2$-agonist

Figure 2. Barplots with error bars on the posterior median (and 95\% credible interval) of the absolute risk of chronic obstructive pulmonary disease (COPD) exacerbation for each intervention in the network [13] under the assumption of transitive risks. Each bar indicates the number of COPD exacerbations per 100 participants who took the intervention. Placebo was the reference intervention in the network. We considered an underlying risk $p_{A}=0.39$ for placebo to obtain the absolute risks for the remaining interventions in the network. ICS, inhaled corticosteroid; LABA, long-acting $\beta 2$-agonist

Figure 3. Plot $\mathrm{A}$ is a barplot on the total required sample size for a future trial on tiotropium versus LABA after adjusting for missing outcome data (MOD) in three different levels of evidence: individual trials, pairwise and network meta-analysis. The horizontal black line refers to the unadjusted total required sample size. On each bar, the percentage refers to the corresponding percentage inflation in the total required sample size when MOD are incorporated in the sample size calculation. Plot B is a barplot with heaped bars on the predicted number of MOD per 100 participants for each intervention of the target comparison in three different levels of evidence. LABA, long-acting $\beta 2$-agonist 


\section{Supplementary files}

Additional file 1: Supplementary Tables (DOCX 39 KB)

Additional file 2: Technical details (DOCX $36 \mathrm{~KB}$ ) 


\section{Figures}

A)

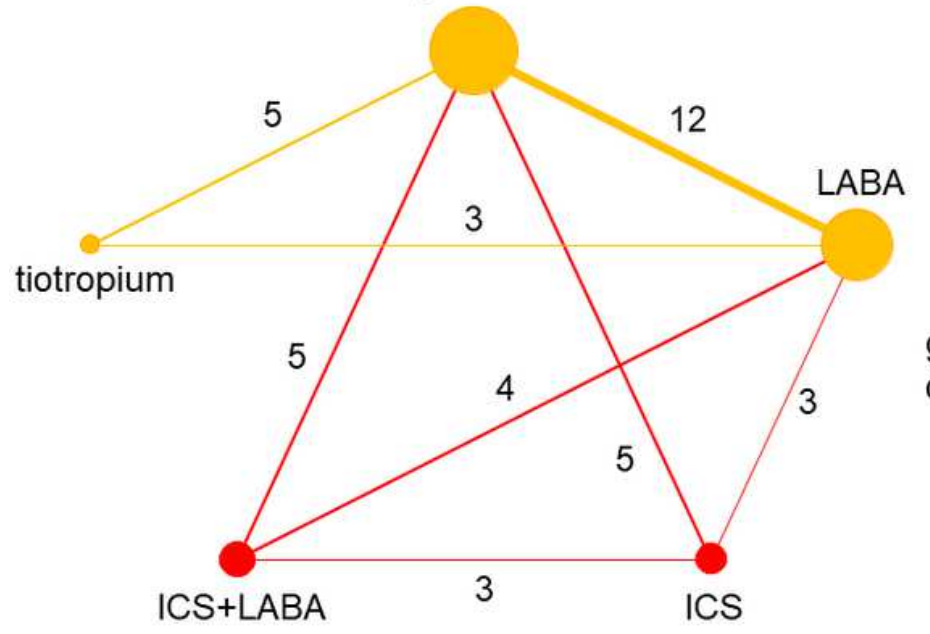

$\square \leq 5$ (low)

$\square(5-20]$ (moderate)

$\square>20$ (high)
B)

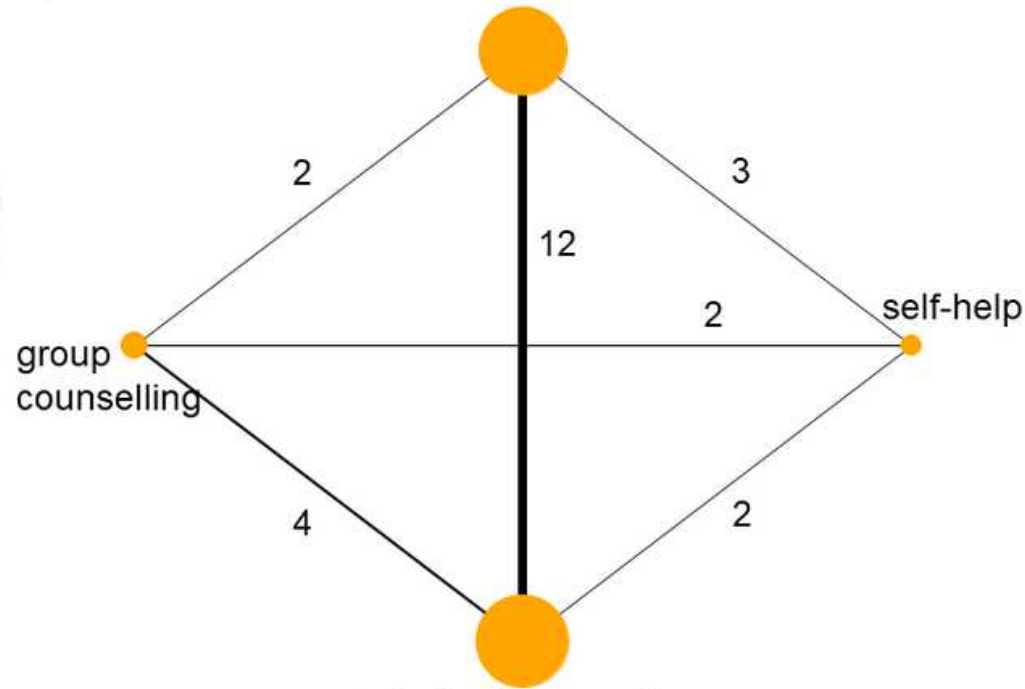

individual counselling

\section{Figure 1}

A network of interventions for the maintenance treatment of the exacerbations of chronic obstructive pulmonary disease (network A) [13]. The different colours in the legend refer to the median percentage of missing outcome data across the trials. A network of four types of counselling for smoking cessation (network B) [19]. In both networks, the size of the nodes is proportional to the number of direct treatment comparisons that include that node, and the thickness of the links is proportional to the number of trials investigating the corresponding comparisons. ICS, inhaled corticosteroid; LABA, long-acting $\beta 2$-agonist 


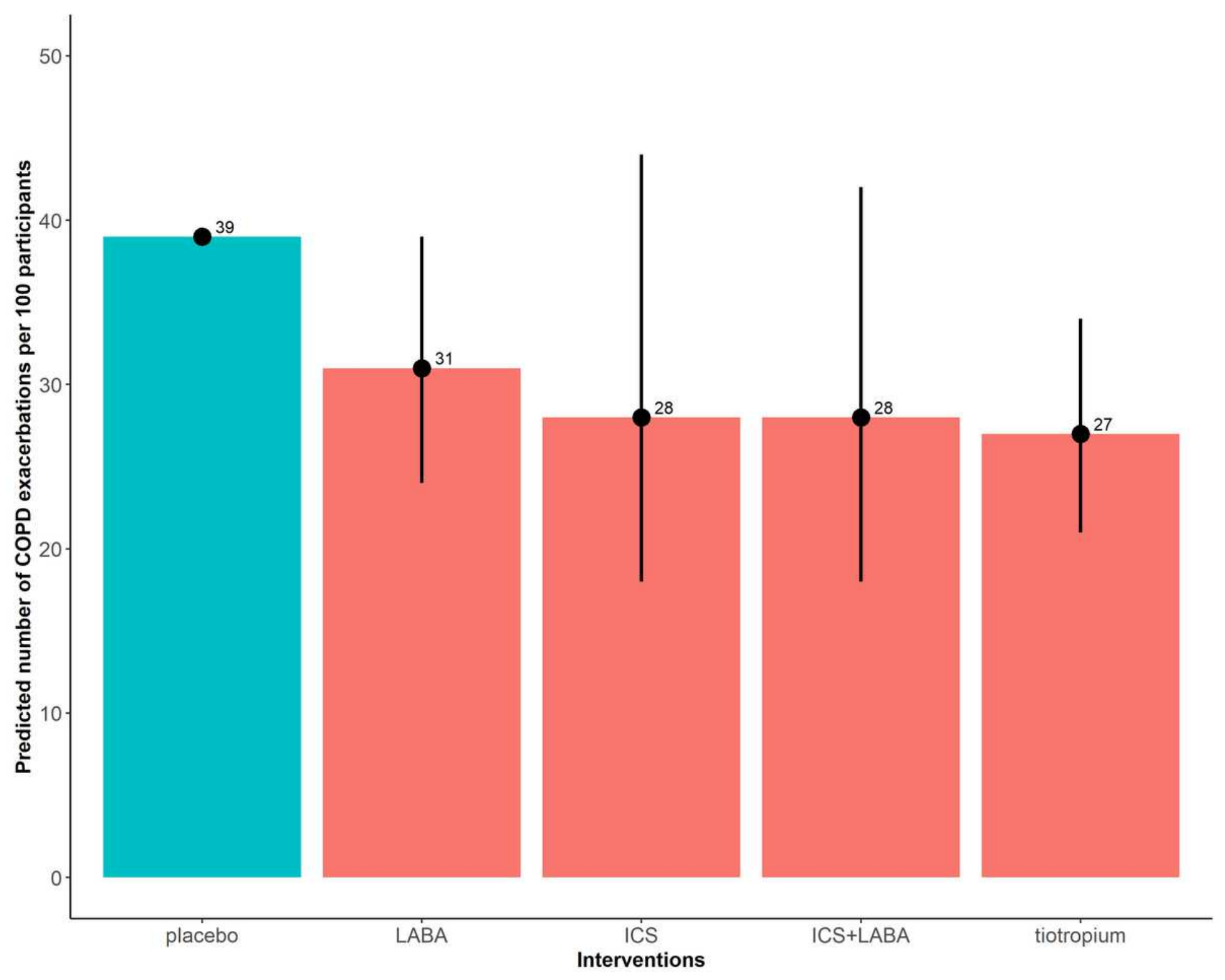

Figure 2

Barplots with error bars on the posterior median (and 95\% credible interval) of the absolute risk of chronic obstructive pulmonary disease (COPD) exacerbation for each intervention in the network [13] under the assumption of transitive risks. Each bar indicates the number of COPD exacerbations per 100 participants who took the intervention. Placebo was the reference intervention in the network. We considered an underlying risk $p_{-} A=0.39$ for placebo to obtain the absolute risks for the remaining interventions in the network. ICS, inhaled corticosteroid; LABA, long-acting $\beta 2$-agonist 

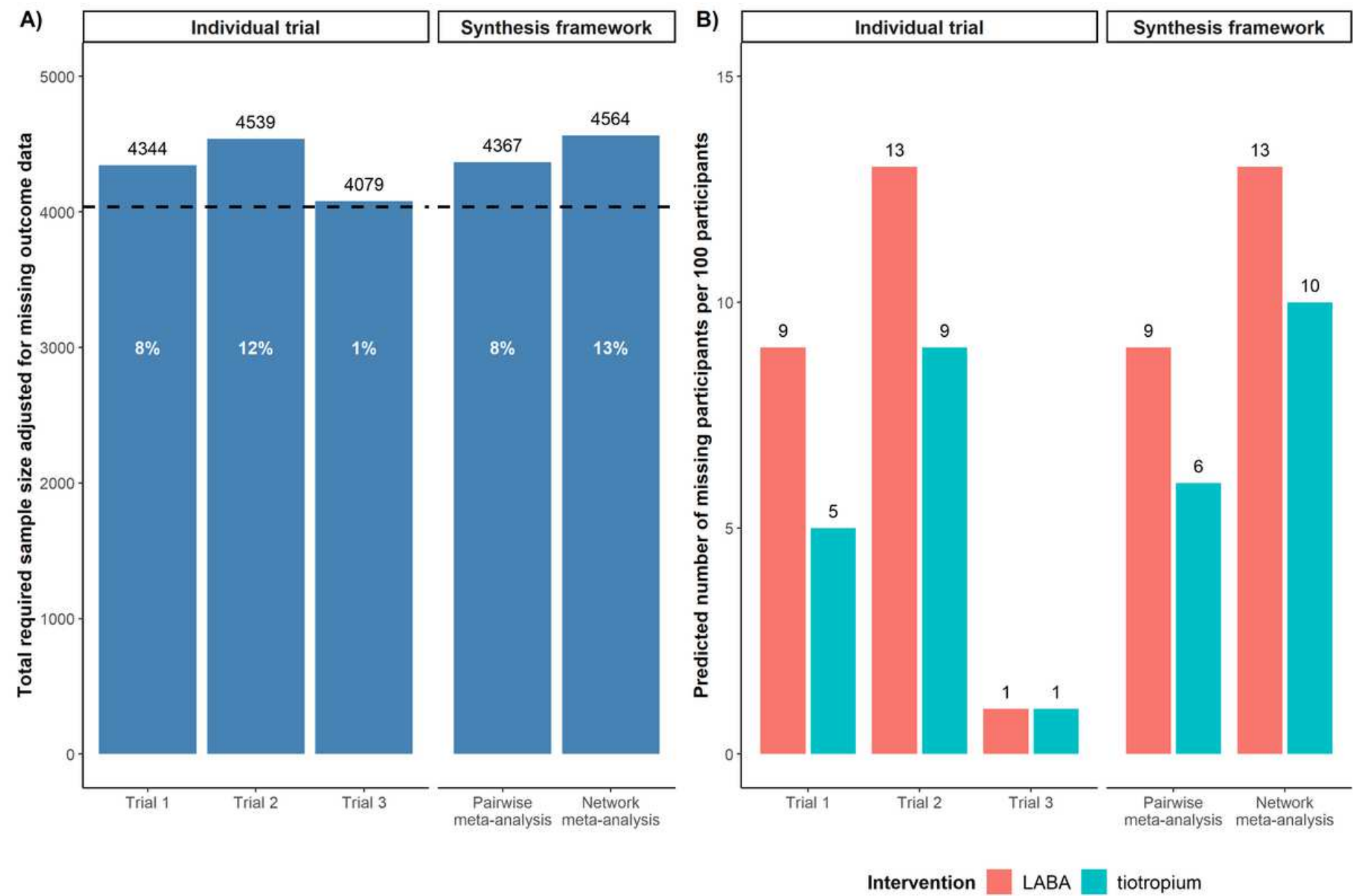

\section{Figure 3}

Plot $A$ is a barplot on the total required sample size for a future trial on tiotropium versus LABA after adjusting for missing outcome data (MOD) in three different levels of evidence: individual trials, pairwise and network meta-analysis. The horizontal black line refers to the unadjusted total required sample size. On each bar, the percentage refers to the corresponding percentage inflation in the total required sample size when MOD are incorporated in the sample size calculation. Plot $B$ is a barplot with heaped bars on the predicted number of MOD per 100 participants for each intervention of the target comparison in three different levels of evidence. LABA, long-acting $\beta 2$-agonist

\section{Supplementary Files}

This is a list of supplementary files associated with this preprint. Click to download.

- Additionalfile1SupplementaryTables.docx

- Additionalfile2Technicaldetails.docx 